\title{
Análise inferencial dos domínios de qualidade de vida em universitários proprietários e não proprietários de cães de estimação
}

\author{
Inferential domains analysis of quality of life among undergraduate owners and non- \\ owners of pet dogs
}

Análisis inferencial de los dominios de calidad de vida en universitarios propietarios y no propietarios de canes de estimación

Francisco José Rivera Pinedo ${ }^{1 *}$, José Bonifácio de Araújo Júnior².

\section{RESUMO}

Objetivo: $O$ presente trabalho procura comparar a qualidade de vida de estudantes de ensino superior proprietários e não proprietários de cachorro de estimação. Metodologia: Por meio de psicometria buscamos reconhecer possíveis efeitos gerados pela interação com cães de estimação. Aplicamos o instrumento de análise da qualidade de vida da Organização Mundial da Saúde (WHOQOL-bref) em 257 estudantes de duas instituições particulares de ensino superior do Distrito Federal. Resultados: Os resultados mostram um ganho no domínio Psicológico (95\% de significância) de estudantes proprietários de cães de estimação e maiores índices dos indicadores de estresse em pessoas não proprietárias. $O$ nosso trabalho também mostra diferenças notáveis entre grupos de homens e mulheres e de Adultos e Jovens, sugerindo diferenças importantes nos projetos de vida perseguidos por cada grupo. Conclusões: Proprietários de cachorro de estimação apresentam escores no domínio Psicológico significativamente maiores que os não proprietários.

Palavras-chave: Apoio social, Estresse psicológico, Qualidade de vida, Relações interpessoais, Psicometria.

\section{ABSTRACT}

Objective: The present work seeks to compare the quality of life among undergraduate owners and nonowners of pet dogs. Methodology: Using psychometrics we seek to recognize possible effects generated by the interaction with estimation dogs. We used the World Health Organization quality of life analysis tool (WHOQOL-bref) in 257 undergraduates from two private institutions in the Federal District, Brazil. Results: The results show an increase in the psychological domain (95\% of significance) of people who own a pet dog and higher indexes of stress indicators in people who do not acquire a dog. Our work also shows differences between men and women and adults and young people suggesting differences in the life projects pursued by each group. Conclusions: Pet dog owners present significantly higher psychological scores than non-pet owners.

Key words: Social support, Psychological stress, Quality of life, Interpersonal relations, Psychometrics.

\section{RESUMEN}

Objetivo: El presente trabajo busca comparar la calidade de vida de estudiantes de educación superior que sean proprietarios y no propietarios de canes de estimação. Metodología: Usando psicometría buscamos reconocer posibles efectos generados por la interacción con canes de estimación. Aplicamos el instrumento para análisis de calidad de vida de la Organización Mundial de la Salud (WHOQOL-bref) en 257 estudiantes universitarios de dos instituciones privadas del Distrito Federal. Resultados: Los resultados indican un aumento del dominio Psicológico (95\% de significancia) en estudiantes con canes de estimación y más altos

\footnotetext{
${ }^{1}$ Instituto Federal Goiano (IF Goiano), Rio Verde - Goiás. *E-mail: fjrpinedo@gmail.com

2 Universidade de Brasília (UnB), Brasília - Distrito Federal.
} 
índices de indicadores de estrés en personas que no poseen canes. Nuestro trabajo también muestra diferencias entre los grupos de hombres y mujeres y de adultos y jóvenes, sugiriendo la existencia de diferencias importantes en sus proyectos de vida. Conclusiones: Propietarios de canes de estimación mostraron puntajes significativamente más altos, en el dominio Psicológico, que los no propietarios.

Palabras clave: Apoyo social, Estrés psicológico, Calidad de vida, Relaciones interpersonales, Psicometría.

\section{INTRODUÇÃO}

Não poder se sustentar sozinho, insegurança quanto ao futuro ou não conseguir trabalhar na área de estudo são as três maiores preocupações profissionais para $60 \%$ de dois mil estudantes universitários pesquisados em São Paulo e Brasília e para $50 \%$ deles a violência, as drogas e o desemprego são as três maiores preocupações fundamentais. Se associarmos esses dados com as prevalências de depressão e ansiedade mostradas, em outra pesquisa, por $30 \%$ de quase 500 estudantes no Nordeste brasileiro e com a ideação suicida apresentada por $10 \%$ de mais de 600 universitários pesquisados em Mato Grosso, poderemos compreender que existem graves dificuldades na juventude brasileira que precisam ser acompanhadas e abordadas (DWYER T, et al., 2016; LEÃO AM, et al., 2018; SANTOS HGB, et al., 2017).

A qualidade de vida é um tema muito discutido em todas as esferas sociais, em pesquisa e nas instâncias do governo, é também um assunto muito estudado em diferentes grupos de pessoas com problemas de saúde física e mental e em idosos, entretanto, são poucas as publicações que abordam esse tipo de estudo em pessoas trabalhadoras comuns e estudantes (SOTHORNWIT J, et al., 2018; HAJEK A, et al., 2018; BEZERRA MLO, et al., 2018).

A melhoria da qualidade de vida é crítica para um conveniente desenvolvimento social e convívio saudável entre as pessoas. Diversas atitudes pessoais e políticas públicas podem ser adotadas com a finalidade de promover saúde, conforto, lazer e, em consequência, qualidade de vida, contudo, alguns importantes empecilhos que atentam contra o bem-estar das pessoas podem ser encontrados na própria cultura das pessoas e das empresas onde trabalham (HIRATA H, 2011; MENDES AD e AZEVÊDO PH, 2010).

O International Stress Management Association, uma organização internacional para a pesquisa do estresse no mundo, aponta o Brasil como o segundo país com maior estresse no mundo. Os efeitos do estresse incluem comportamento provocativo, isolamento, ansiedade e depressão, além de transtornos na atividade sexual e uma cascata de outros eventos fisiológicos que comprometem de forma importante os sistemas nervoso, endócrino, imunológico e cardiovascular (SCHNEIDERMAN N, et al., 2005; MATSUHISA F, et al., 2014).

As relações entre o estresse e a perda de qualidade de vida em pessoas sem doença crônica é inegável e deveriam ser consideradas nos programas de saúde ocupacional e nos cuidados pessoais que todo cidadão deveria zelar (MORAES LFR, et al., 2003; MARQUES AL, et al., 2003; PAIVA KCM, COUTO JH, 2008).

A melhoria da qualidade de vida nas pessoas passa por mudanças comportamentais e culturais, entretanto é difícil definir como e onde isso poderia ocorrer, pois se trata de um assunto de índole psicossocial e muito particular de cada pessoa. Contudo, o uso atual da mídia como meio de interação social poderia ser útil, também, como catalizador para a modificação do comportamento com a finalidade de promover uma melhoria nas pessoas (HJARVARD S, 2011).

Ações diretas ou indiretas, públicas ou particulares que promovam a saúde física, mental e a qualidade de vida precisam ser adotadas de forma consciente e criteriosa e essas mudanças incluem hábitos e relacionamentos particulares no ambiente do lar, onde nasce a maioria das alterações comportamentais como respostas de adaptação e interação (SPARRENBERGER F, et al., 2003; MARTEAU TM, et al., 2012; BROOM DM, 1998). A interação entre o homem e o seu animal de estimação parece ser muito peculiar e deve promover alterações comportamentais importantes embora não sejam bem reconhecidas. Inegáveis são os diversos efeitos estimulados pelo animal de estimação na maioria dos seus proprietários e que incluem alívio 
de tensões, bom humor, maior sensação de utilidade e afeto, promoção da sociabilidade, entre outros (FUCHS H, 1987; McNICHOLAS J, et al., 2005; OLIVEIRA GR, et al., 2016).

A relação afetiva estabelecida entre a pessoa e o seu animal de estimação parece ser mais importante que a própria estimulação da atividade física, muito documentada e promovida principalmente por cachorros de estimação. A importância da interação afetiva é evidenciada em diversas publicações, com destaque para o trabalho publicado em 1980 com mais de 90 pessoas afetadas por infarto do miocárdio ou angina de peito, das quais o grupo que não possuía animal de estimação mostrou uma sobrevivência anual de $72 \%$ contra 94\% dos pacientes que possuíam animal de estimação dos quais a grande maioria possuíam cachorro como único animal de estimação (FRIEDMANN E, et al., 1980; LEVINE GN, et al., 2013; KATHIE MC, et al., 2007).

Os cachorros são os animais que mais interagem com os seus proprietários e promovem um maior apoio social e motivação para atividade física. Foi reconhecido que o benefício na redução do risco cardiovascular pode-se dever a ambas as estimulações social e física que os cachorros promovem (MUBANGA M, et al., 2017; CHRISTIAN HE, et al., 2013).

Também foi identificada a participação de cachorros na obtenção de valores estatisticamente significativos de recuperação de pessoas com outras doenças como depressão e ansiedade (MULDOON AL, et al., 2017; BARKER SB, DAWSON KS, 1998; CREAGAN ET, 2015).

O presente trabalho busca identificar diversos benefícios nas relações sociais, interação com o meio ambiente, status psicológico e condição física, contemplados nos domínios do instrumento de Qualidade de Vida da Organização Mundial da Saúde (WHOQOL-bref), benefícios que os proprietários podem adquirir pela interação com os seus cachorros de estimação.

\section{MÉTODOS}

O presente trabalho trata de uma análise descritiva-inferencial da qualidade de vida de 257 estudantes de graduação de duas instituições de ensino superior do Distrito Federal, Brasil. Os estudantes pertenceram a diferentes cursos de graduação das áreas das Ciências da Saúde, Ciências Sociais Aplicadas e Ciências Humanas.

Foram excluídos alunos do curso de Medicina Veterinária. Para a análise de qualidade de vida foi utilizado o instrumento abreviado de Qualidade de Vida da Organização Mundial da Saúde (World Health Organization Quality of Life, WHOQOL-bref) o qual é constituído de 26 perguntas escalonadas.

Os dados foram organizados em agrupamentos segundo a idade (Adulto ou Jovem), sexo (homem ou mulher) e em subgrupos de Proprietários ou Não Proprietários pela presença ou não de cachorro de estimação em seus lares.

Para o presente trabalho foram consideradas pessoas Jovens aos participantes com até 26 anos de idade e, pessoas Adultas àqueles acima de 26 anos de idade.

As respostas ao instrumento WHOQOL-Bref adotaram valores de 1 a 5 . As respostas de número 3, 4 e 26 tiveram os seus valores recodificados, como indicado no instrumento utilizado ([1=5]; $[2=4] ;[3=3] ;[4=2]$; $[5=1])$.

Os dados dos participantes e os valores das respostas a cada uma das perguntas foram organizados e um arquivo de Excel (Microsoft $(\mathrm{B})$. Para o processamento dos dados e as análises estatísticas das respostas foi utilizada a linguagem de programação de Python 3.6.4.

Para identificar a interferência pela presença ou não de cachorro de estimação nos valores de qualidade de vida, foi aplicada a estatística inferencial para análise de variância (ANOVA) e o teste de Fisher. Os procedimentos estatísticos aplicados foram armazenados na plataforma de hospedagem GitHub e podem ser acessados no endereço: https://github.com/HoltechHard/study_quality_life. 
O projeto correspondente ao presente trabalho foi submetido a um comitê de ética em pesquisa via Plataforma Brasil, a base unificada de registros de pesquisas e foi aprovado sob o número de Certificado de Apresentação para Apreciação Ética (CAAE): 51129915.3.0000.5595.

\section{RESULTADOS}

O conceito de qualidade de vida do instrumento WHOQOL-bref é formado a partir das 26 perguntas que estão agrupadas em quatro grandes esferas da vida cotidiana chamadas domínios Psicológico, Relações Sociais, Meio Ambiente e Físico (Figura 1).

Figura 1- Esquema dos domínios e itens do instrumento de qualidade de vida WHOQOL-bref.

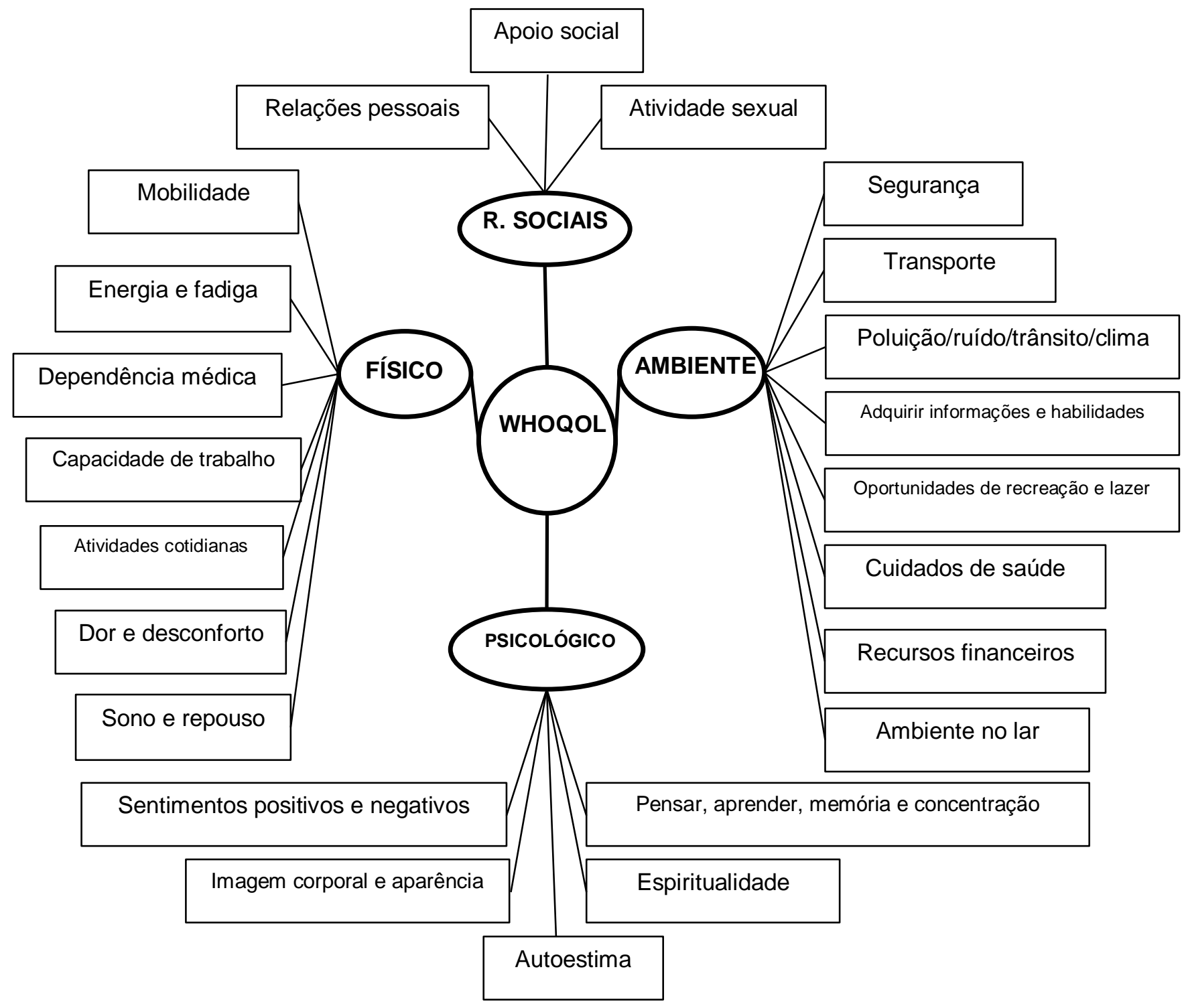

Fonte: Dados da pesquisa, 2019.

O instrumento WHOQOL-bref foi aplicado a 257 alunos de ensino superior e os seus dados foram organizados em nove agrupamentos segundo critérios de sexo, faixa etária e posse de cachorro de estimação. A discrepância entre o número total e o somatório de proprietários e não proprietários, em alguns grupos, ocorreu pela falta de alguns dados pessoais nas respostas preenchidas pelos participantes (Figura 2). 
Figura 2 - Agrupamentos e número de participantes da pesquisa.

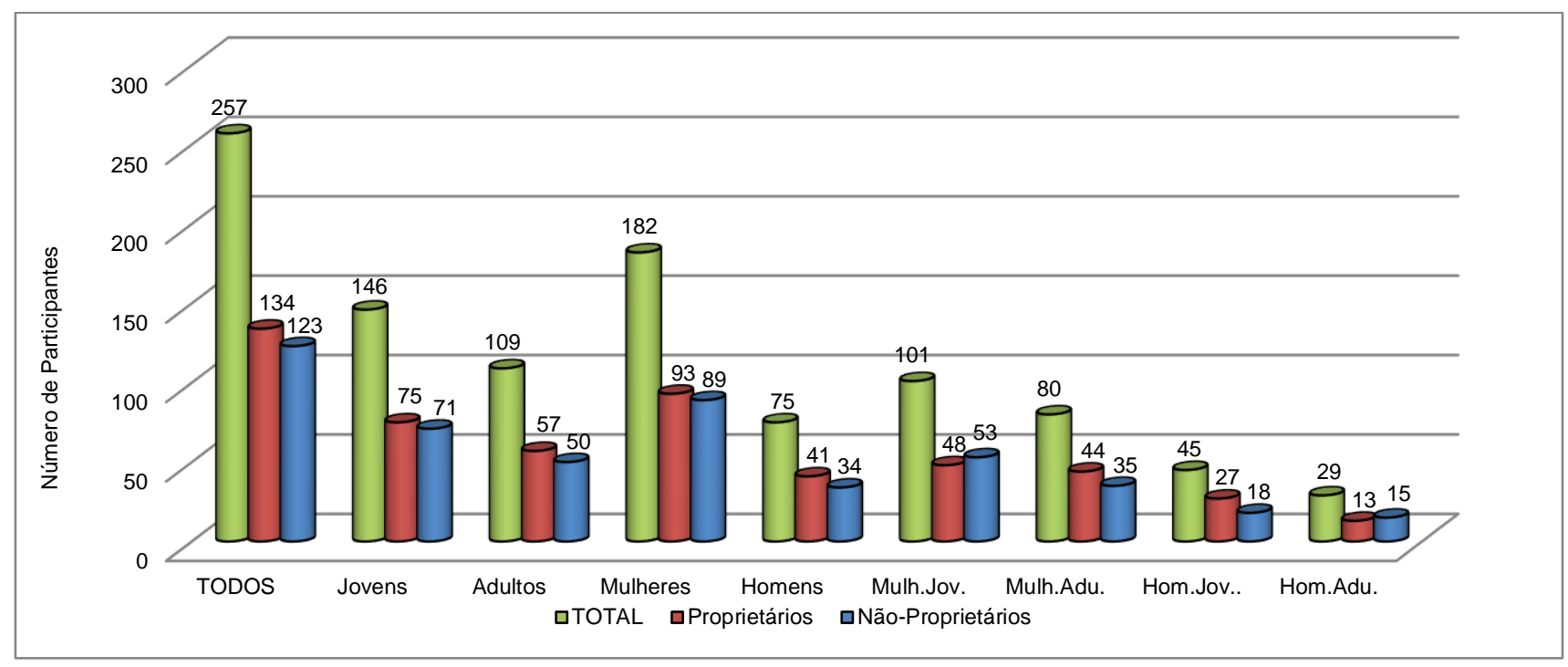

Legenda: Mulh.Jov= mulheres jovens; Mulh.Adu= mulheres adultas; Hom.Adu= homens adultos; Hom.Jov= homens jovens.

Fonte: Dados da pesquisa, 2019.

Embora os valores psicométricos não apontem uma relação entre a presença de cachorro e o desempenho dos domínios Relações Sociais, Ambiente e Físico, no presente trabalho, avaliamos a possibilidade de os valores estatisticamente não significativos representarem diferenças fisiológicos ou comportamentais importantes. Nesse sentido, utilizamos os valores do teste de Fisher que não atingiram o nível de significância e obtivemos um retrato das diferenças entre proprietários e não proprietários (Figura 3).

Os resultados apontaram que os proprietários possuem importantes ganhos psicométricos nos grupos de adultos e mulheres adultas (domínio Relações Sociais) e nos grupos de homens e homens jovens (domínio Físico) quando consideramos valores do teste-F superiores a 1 (um) (Figura 3).

Figura 3 - Ganho comparativo em Qualidade de Vida entre Proprietários e Não proprietários.

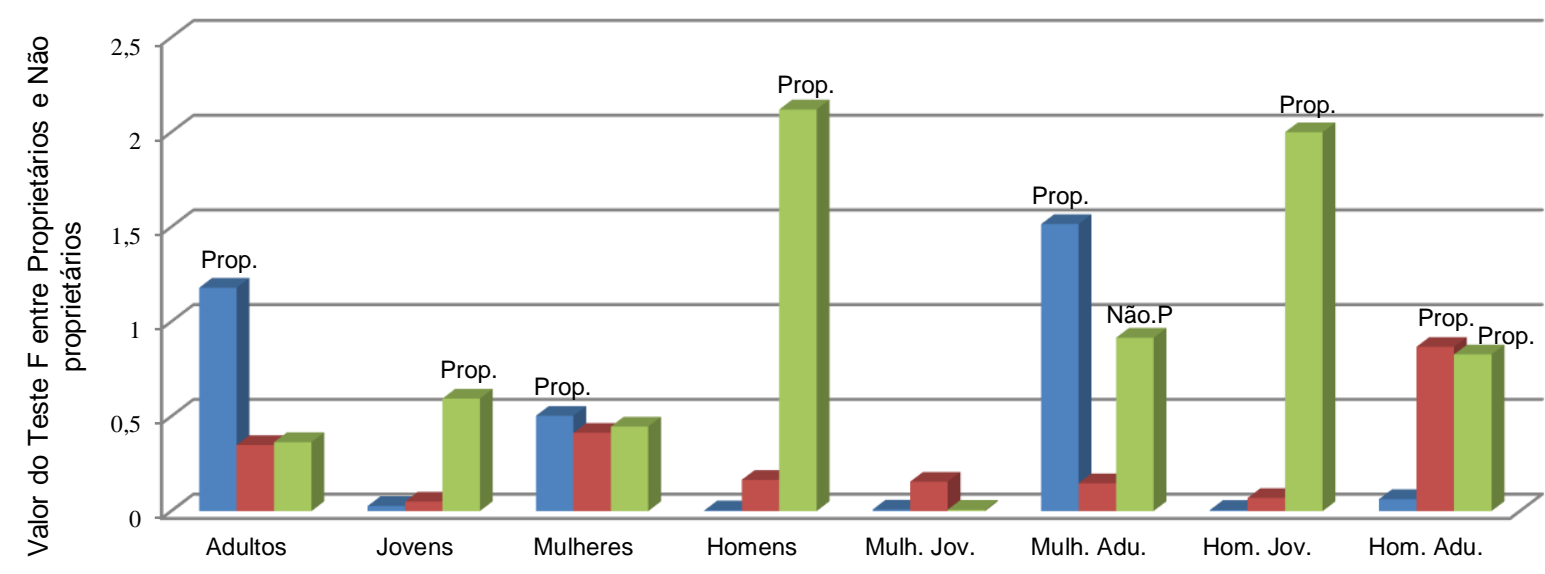

Grupos de Proprietários vs Não proprietários

$$
\llbracket \mathrm{d} \_r s \backsim d \_a m b \quad d \_f i s
$$

Legenda: Prop.= proprietários. Não.P.= não proprietários. d_rs= domínio relações sociais; d_amb= domínio meio ambiente; d_fis=domínio físico. Fonte: Dados da pesquisa, 2019. 
Para cada um dos subgrupos de proprietários e não proprietários foram realizadas análises que incluíram a média aritmética e o seu valor de dispersão ou variância amostral em cada domínio. Para obtenção da significância das diferenças entre os escores dos domínios de qualidade de vida de proprietários versus não proprietários foi aplicada a análise de variância (ANOVA) e obtido o valor do teste de Fischer (Tabela 1).

Ao compararmos proprietários com não proprietários totais (primeiro grupo da Tabela 1) identificamos um melhor desempenho (95\% de significância), apenas no domínio Psicológico e a favor dos participantes proprietários de cachorro de estimação, sendo que, as mulheres jovens proprietárias formam o principal subgrupo responsável pelo melhor desempenho do domínio Psicológico visto nos proprietários totais. Nem o grupo de adultos nem o grupo de homens jovens não apresentam diferenças significativas nos quatro domínios pela presença ou ausência de cachorro de estimação.

Tabela 1 - Análise de Variancia de Proprietários versus Não Proprietários.

\begin{tabular}{|c|c|c|c|c|c|}
\hline \multirow{2}{*}{ Grupos } & \multirow{2}{*}{ Média de Idade } & \multicolumn{4}{|c|}{ Médias dos domínios de qualidade de Vida (DP) } \\
\hline & & Psicológico & Relações sociais & Ambiente & Físico \\
\hline \multicolumn{6}{|c|}{ 1- TODOS } \\
\hline Proprietários & 27,5 & $3,92(0,57)$ & $3,85(0,80)$ & $3,18(0,61)$ & $3,78(0,64)$ \\
\hline Não-Proprietários & 26,1 & $3,78(0,53)$ & $3,79(0,67)$ & $3,13(0,56)$ & $3,77(0,54)$ \\
\hline Teste F & & $4,094^{*}$ & 0,391 & 0,557 & 0,02 \\
\hline \multicolumn{6}{|c|}{ 2- ADULTOS (+ 26 anos) } \\
\hline Proprietários & 34,9 & $3,87(0,66)$ & $3,80(0,85)$ & $3,19(0,62)$ & $3,76(0,72)$ \\
\hline Não-Proprietários & 33,3 & $3,77(0,58)$ & $3,63(0,74)$ & $3,13(0,52)$ & $3,83(0,61)$ \\
\hline Teste F & & 0,712 & 1,179 & 0,349 & 0,364 \\
\hline \multicolumn{6}{|c|}{ 3- JOVENS (até 26 anos) } \\
\hline Proprietários & 21,9 & $3,96(0,51)$ & $3,88(0,77)$ & $3,15(0,60)$ & $3,78(0,59)$ \\
\hline Não-Proprietários & 21,1 & $3,78(0,49)$ & $3,90(0,61)$ & $3,12(0,60)$ & $3,71(0,49)$ \\
\hline \multicolumn{6}{|c|}{ 4- MULHERES } \\
\hline Proprietárias & 27,7 & $3,92(0,58)$ & $3,87(0,79)$ & $3,18(0,62)$ & $3,73(0,68)$ \\
\hline Não-Proprietárias & 25,8 & $3,78(0,55)$ & $3,79(0,71)$ & $3,13(0,54)$ & $3,80(0,54)$ \\
\hline Teste F & & $\begin{array}{l}\text { 2,612 } \\
\text { 5- HOME }\end{array}$ & 0,503 & 0,413 & 0,446 \\
\hline Proprietário & 26,9 & $3,93(0,56)$ & $3,81(0,84)$ & $3,18(0,62)$ & $3,89(0,54)$ \\
\hline Não-Proprietários & 27 & $3,79(0,47)$ & $3,80(0,58)$ & $3,12(0,64)$ & $3,70(0,56)$ \\
\hline Teste F & & 1,48 & 0,004 & 0,165 & 2,12 \\
\hline \multicolumn{6}{|c|}{ 6- MULHERES JOVENS } \\
\hline Proprietárias & 21,9 & $3,98(0,47)$ & $3,90(0,76)$ & $3,14(0,60)$ & $3,71(0,62)$ \\
\hline Não-Proprietárias & 20,8 & $3,78(0,50)$ & $3,91(0,62)$ & $3,09(0,55)$ & $3,72(0,48)$ \\
\hline Teste $\mathrm{F}$ & & $4,371^{*}$ & 0,008 & 0,155 & 0,007 \\
\hline \multicolumn{6}{|c|}{ 7- MULHERES ADULTAS } \\
\hline Proprietárias & 34,1 & $3,84(0,68)$ & $3,83(0,83)$ & $3,22(0,63)$ & $3,74(0,75)$ \\
\hline Não-Proprietárias & 33,4 & $3,77(0,63)$ & $3,61(0,81)$ & $3,18(0,53)$ & $3,91(0,62)$ \\
\hline Teste F & & 0,279 & 1,515 & 0,147 & 0,915 \\
\hline \multicolumn{6}{|c|}{ 8- HOMENS JOVENS } \\
\hline Proprietários & 21.8 & $3,92(0,58)$ & $3,85(0,80)$ & $3,16(0,60)$ & $3,90(0,50)$ \\
\hline Não-Proprietários & 21,8 & $3,79(0,46)$ & $3,87(0,59)$ & $3,21(0,75)$ & $3,68(0,54)$ \\
\hline Teste $\mathrm{F}$ & & 0,653 & 0,006 & 0,069 & 20 \\
\hline \multicolumn{6}{|c|}{ 9- HOMENS ADULTOS } \\
\hline Proprietários & 37,4 & $3,95(0,57)$ & $3,67(0,93)$ & $3,11(0,59)$ & $3,81(0,61)$ \\
\hline Não-Proprietários & 33,2 & $3,75(0,49)$ & $3,67(0,54)$ & $3,00(0,51)$ & $3,67(0,56)$ \\
\hline Teste $\mathrm{F}$ & & 1,228 & 0,063 & 0,867 & 0,827 \\
\hline
\end{tabular}

* 95\% de significância. Fonte: Dados da pesquisa, 2019.

O conjunto 1 é formado basicamente de adultos e mostram comportamentos de correlação entre domínios muito semelhantes entre si, diferentes ao Conjunto 2 que é formado basicamente de jovens e possuem correlações muito variáveis e sem um padrão definido. 
Nós também verificamos se haveriam diferenças importantes na forma como os domínios se interrelacionam dentro de cada subgrupo e se a presença de cachorro de estimação poderia alterar essas interrelações e, com isso, alterar a forma das pessoas perceberem ou interagirem com o seu entorno. Para isso utilizamos os valores dos domínios de cada subgrupo e obtivemos a correlação de Pearson e, a partir dos resultados, foram gerados dois conjuntos (Figura 4).

Os conjuntos destacam-se pela maior diferença de correlação entre os domínios Psicológico versus físico, e, pela maior semelhança entre os domínios Físico e Ambiente. Os dados também apontam que as mulheres apresentam maior correlação entre os domínios Relações Sociais versus psicológico em comparação com os homens, sem mostrarem efeitos pela posse de cachorro. Já, em mulheres jovens, a presença de cachorro está fortemente associada a uma maior correlação entre os domínios Físico versus Relações Sociais. Nos homens adultos verifica-se que a presença de cachorro está associada a uma substancial redução da correlação entre domínios Psicológico versus físico. Nos homens jovens a presença de cachorro associa-se a uma redução da correlação entre domínios Psicológico versus físico (Figura 4).

Figura 4 - Correlações de Pearson para os dois Conjuntos de Participantes.

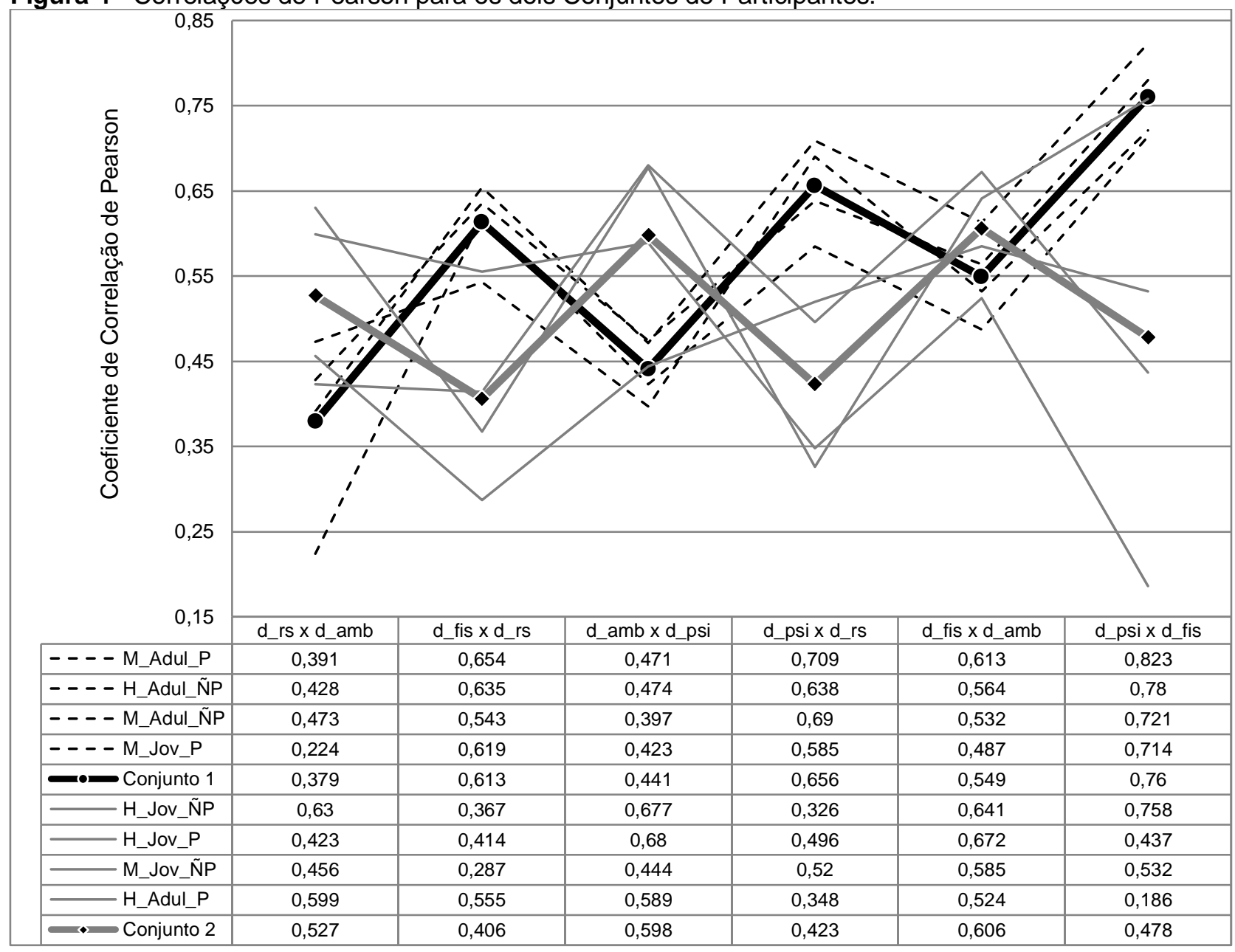

Legenda: d_rs= domínio relações sociais; $d \_a m b=$ domínio meio ambiente; d_fis=domínio físico; $M \_A d u l \_P=$ mulheres adultas proprietárias; $H \_$Adult_ $\tilde{N} P=$ homens adultos não proprietários; $M \_A d u l \_\tilde{N} P=$ mulheres adultas não proprietárias; $M \_J o v \_P=$ mulheres jovens proprietárias; $H \_J o v \_\tilde{N} P=$ homens jovens não proprietários; H_Jov_P=homens jovens proprietários; $M \_J o v \_\tilde{N} P=m u l h e r e s$ jovens não proprietárias; H_Adul_P=homens adultos proprietários.

Fonte: Dados da pesquisa, 2019. 


\section{DISCUSSÃO}

Calais et al (2003) mostraram, em um trabalho com 295 estudantes, que as mulheres jovens, entre 15 a 28 anos, são o grupo de pessoas com a maior incidência de estresse e, no trabalho de Lipp com 1818 pessoas, como destacado por Lipp (2007), as mulheres já eram apontadas em 1996 como o gênero que mais estressa no Brasil. Esses resultados são úteis para compreender a maior associação entre a presença de cachorro e o aumento da qualidade do domínio Psicológico observado nas mulheres jovens participantes do nosso trabalho.

Analisando com maior detalhe as respostas do grupo de mulheres jovens encontramos que, diante da pergunta 7 (WHOQOL_bref) que aborda a capacidade de concentração, 38\% das não proprietárias contra $54 \%$ das proprietárias afirmam ter "bastante" ou "extrema" capacidade de concentração e para a pergunta 26 , que indaga a existência de estados de mau humor, desespero, ansiedade e depressão, $55 \%$ das não proprietárias, contra $73 \%$ das correspondentes mulheres jovens proprietárias, afirmam que "nunca" ou "algumas vezes" passaram por esses estados. Essas respostas, aos itens 7 e 26, apontam para uma menor frequência de sintomas de estresse em mulheres jovens proprietárias de cachorro de estimação. Consideramos também um destaque o fato das mulheres jovens proprietárias apresentarem uma característica que é comum aos adultos do nosso trabalho e refere-se à alta correlação entre os domínios Físico versus Relações Sociais e, se atentarmos para o elevado escore do domínio Relações Sociais encontrado nas mulheres jovens (como nos homens jovens) compreenderemos que a elevada correlação mencionada participaria de forma importante no melhor desempenho do domínio Físico neste subgrupo.

Os dados psicométricos da figura 3 sugerem uma melhora, estatisticamente não significativa, do domínio Relações Sociais na presença de cachorro em mulheres adultas. Essa melhora pode ser corroborada com a maior percepção de apoio social (pergunta 22 do WHOQOL-bref) indicada nas respostas "satisfeito" ou "muito satisfeito" de $75 \%$ das mulheres adultas proprietárias em comparação ao $43 \%$ das correspondentes não proprietárias.

A melhoria no domínio Relações Sociais e, especificamente, na percepção de apoio social recebido no subgrupo de mulheres adultas proprietárias pode estar associada à proposta apresentada por McNicholas et al. (2005) que consideram que o animal de estimação atuaria como um catalizador social favorecendo o contato entre as pessoas e, ao mesmo tempo, fornecendo um suporte afetivo e companhia que condicionariam favoravelmente para a melhora das relações sociais.

Por outro lado, Feldmann et al. (2008) ao analisar a percepção de apoio social de 300 estudantes universitários encontrou correlação positiva com a saúde mental e negativa com o estresse acadêmico com o que conseguimos inferir que a presença de cachorro em mulheres adultas poderá estar também associada tanto com a saúde mental quanto com o desempenho acadêmico.

A análise das respostas relativas à manifestação de estados de mau humor, desespero, ansiedade e depressão (pergunta 26 do WHOQOL-bref) indica que a população adulta é mais homogênea e menos vulnerável que a população jovem. Na população adulta pesquisada $82,8 \%$ tem respostas favoráveis indicando que "Nunca" ou só "Algumas vezes" passaram por esses estados diante da população jovem que se apresenta dividida e onde $73,3 \%$ dos proprietários tiveram respostas favoráveis contra apenas $54,9 \%$ dos jovens não proprietários. As maiores dificuldades destacadas pelos jovens não proprietários podem estar associadas a uma conjuntura de dependência familiar por se tratar de estudantes.

Já o melhor desempenho dos jovens proprietários pode ser consequência de uma postura psicologicamente diferente à dos jovens não proprietários diante das mesmas circunstâncias. Circunstâncias adversas enfrentadas por jovens também são observadas em um estudo em Portugal realizado por Silva MG e Costa ME (2005) com 511 estudantes universitários da mesma faixa etária (17 e 26 anos) onde se verificou que os jovens com estilos de vinculação insegura aos pais, por preocupação ou amedrontamento que podem 
se instalar por relações de superproteção ou rejeição, apresentam maior risco a desenvolver ansiedade tornando o indivíduo mais vulnerável, frágil e dependente de figuras protetoras. Estas situações precisariam ser analisadas na população jovem brasileira tendo em vista o seu impacto na autoconfiança e visão tanto de si próprio quanto do mundo ao redor, os quais são determinantes do sucesso profissional.

Segundo dados de vitimização do Instituto Brasileiro de Geografia e Estatística (IBGE, 2010) 51\% dos brasileiros adultos entre 25 a 59 anos sentem-se seguros na cidade onde moram, um valor muito próximo ao obtido no nosso trabalho onde apenas $56 \%$ dos adultos (acima de 26 anos) afirmam se sentir "Bastante" ou "Extremamente" seguros (pergunta 8 do WHOQOL-bref).

Já nos homens adultos proprietários esse valor sobe para 69\% e para os homens adultos não proprietários o valor cai para apenas $20 \%$. O valor de $20 \%$ é muito baixo inclusive se comparado ao $43 \%$ da população do Distrito Federal (de ambos os sexos) que afirma se sentir segura na capital do país (IBGE, 2010).

Apesar do número baixo de homens adultos participantes, acreditamos ser muito relevante considerar os nossos achados e cogitar a possibilidade de a presença de cachorros de estimação estar favorecendo uma maior percepção de segurança na população adulta masculina mesmo quando o cachorro não for adquirido para tal finalidade.

Finalmente, sendo os cachorros animais de relevância social e capazes de modular o comportamento humano, cremos ser necessária a identificação das formas como as pessoas interagem com eles e avaliar se essas formas de interação estariam associadas a características comportamentais particulares e socialmente relevantes.

\section{CONCLUSÕES}

O teste exato de Fisher aplicado no nosso trabalho aponta para a existência de um maior desenvolvimento do domínio Psicológico em estudantes que possuem cachorro de estimação com destaque para as mulheres jovens (95\% de significância). Os nossos dados psicométricos também identificaram maior desenvolvimento dos domínios Físico em homens proprietários e Relações Sociais em mulheres adultas proprietárias quando comparados aos correspondentes não proprietários.

\section{AGRADECIMENTOS}

Agradecemos à professora Ana Angélica Gonçalves Paiva pela confiança depositada nos pesquisadores responsáveis pelo presente trabalho, ao professor Dr. Aparecido Pimentel Ferreira pelo permanente incentivo e pelas importantes ideias para a confecção deste trabalho e aos senhores veterinários José Wilker de Amaral, Agda Regina Melo Silva, Isabela Oliveira Lima e Eslaine da Silva Veiga pelo empenho na compilação de dados e sem os quais a presente pesquisa não teria iniciado.

\section{REFERÊNCIAS}

1. BARKER SB, DAWSON KS. The effects of Animal-Assisted Therapy on anxiety ratings of hospitalized psychiatric patients. Psychiatric Services, 1998; 49(6): 797-802.

2. BEZERRA MLO, SIQUARA GM, ABREU JNS. Relação entre os pensamentos ruminativos e índices de ansiedade e depressão em estudantes de psicologia. Rev Psi Divers Saúde, 2018; 7(2): 235-244.

3. BROOM DM. Welfare, Stress, and the Evolution of Feelings. Advances in the Study of Behavior, 1998; 27: 371-403.

4. CALAIS SL, ANDRADE LMB, LIPP MEN. Diferenças de Sexo e Escolaridade na Manifestação de Stress em Adultos Jovens. Psicologia: Reflexão e Crítica, 2003; 1(2): 257-263.

5. CHRISTIAN HE, WESTGARTH C, BAUMAN A, Richards EA, Rhodes RE, Evenson KR, Mayer JA, Thorpe RJ Jr. Dog Ownership and Physical Activity: A Review of the Evidence. J Phys Act Health, 2013; 10(5): 750-759. 
6. CREAGAN ET, BAUER BA, THOMLEY JMB, et al. Animal-assisted therapy at Mayo Clinic: The time is now. Complement Ther Clin Pract, 2015; 21(2): 101-104.

7. MORAES LFR, MARQUES AL; PORTES PCP. Qualidade de vida no trabalho e estresse ocupacional na Polícia Militar de Minas Gerais. Revista de Psicologia: Saúde Mental e Segurança Pública, 2003; 3: 53-58.

8. OLIVEIRA GR, ICHITANI T, CUNHA MC. Atividade Assistida por Animais: efeitos na comunicação e interação social em ambiente escolar. Distúrbios da Comunicação, 2016; 28(4): 759-763.

9. PAIVA KCM, COUTO JH. Qualidade de vida e estresse gerencial "pós-choque de gestão": o caso da Copasa-MG. Revista de Administração Pública, 2008; 42(6): 1189-1211.

10. DWYER T, ZEN EL, WELLER W, et al. (Organizadores). Jovens universitários em um mundo em transformação: uma pesquisa sino-brasileira. Brasília: Ipea; Pequim: SSAP; 2016; 311 p.

11. FELDMANN L, GONÇALVES L, CHACÓN-PUIGNAU G, et al. Relaciones entre estrés académico, apoyo social, salud mental y rendimiento académico en estudiantes universitarios venezolanos, Bogotá. Universitas Psychologica, 2008; 7(3): 739-751.

12. FRIEDMANN E, KATCHER AH, LYNCH JJ. Animal Companions and One-Year Survival of Patients After Discharge From a Coronary Care Unit. Public Health Reports 1980; 95(4): 307-312.

13. FUCHS H. O animal em casa: Um estudo no sentido de des-velar o significado psicológico do animal de estimação, SP. Tese (Doutorado em Ciências) - Instituto de Psicologia. Universidade de São Paulo, São Paulo, 1987; 186 p.

14. HAJEK A, BRETTSCHNEIDER C, VAN DEN BUSSCHE H et al. Impact of falls on depressive symptoms among the oldest old: Results from the AgeQualiDe study. Int J Geriatr Psychiatry, 2018; 33: 1383-1388.

15. HIRATA H. Tendências recentes da precarização social e do trabalho: Brasil, França, Japão. Caderno CRH, 2011; 24(Supl. 1): 13-20.

16. HJARVARD S. Midiatização: teorizando a mídia como agente de mudança social e cultural. Matrizes, 2012; Janeiro-Junho; 5(2): 53-91.

17. Instituto Brasileiro de Geografia e Estatística (IBGE). Pesquisa Nacional por Amostra de Domicílios. Características da Vitimização e do Acesso à Justiça no Brasil 2009: Brasil/IBGE, Coordenação de Trabalho e Rendimento. Rio de Janeiro: IBGE; 2010.

18. KATHIE MC, GAWLINSKI A, STEERS N. Animal-Assisted Therapy in Patients Hospitalized With Heart Failure. Am J Crit Care, 2007; 16(6): 575-585.

19. LEÃO AM, GOMES IP, FERREIRA MJM, et al. Prevalência e Fatores Associados à Depressão e Ansiedade entre Estudantes Universitários da Área da Saúde de um Grande Centro Urbano do Nordeste do Brasil. Rev. bras. educ. med., 2018; 42 (4): 55 65.

20. LEVINE GN, KAREN ALLEN K, BRAUN LT, et al. Pet Ownership and Cardiovascular Risk: A Scientific Statement From the American Heart Association. Circulation, 2013; June 11; 127(23): 2353-2363.

21. LIPP MEN. Transtorno de Adaptação. Boletim Academia Paulista de Psicologia, 2007, XXVII, 1(7): 72-82.

22. MARQUES AL, MORAIS LFR, PEREIRA LZ, et al. Implicações do treinamento e desenvolvimento de pessoal na qualidade de vida e estresse no trabalho. Revista de Psicologia: Saúde Mental e Segurança Pública, 2003; 3: 43-52.

23. MARTEAU TM, HOLLANDS GJ, FLETCHER PC. Changing Human Behavior to Prevent Disease: The Importance of Targeting Automatic Processes. SCIENCE 2012; 337: 1492-1495.

24. MATSUHISA F, KITAMURA N, SATOH E. Effects of acute and chronic psychological stress on platelet aggregation in mice, Amsterdam. Stress, 2014; 17(2): 186-192.

25. MCNICHOLAS J, GILBEY A, RENNIE A, et al. Pet ownership and human health: a brief review of evidence and issues. BMJ 2005; 331: 1252-1255.

26. MENDES AD, AZEVÊDO PH. Políticas públicas de esporte e lazer \& políticas públicas educacionais. Rev. Bras. Cienc. Esporte, 2010; 32(1):127-142.

27. MUBANGA M, BYBERG L, NOWAK C, et al. Dog ownership and the risk of cardiovascular disease and death - a nationwide cohort study. Scientific Reports 2017; 7: 15821: 1-9.

28. MULDOON AL, KUHNS LM, SUPPLE J, et al. Web-Based Study of Dog Ownership and Depression Among People Living With HIV. JMIR Ment Health, 2017; 4(4):e53: 1-10.

29. SANTOS HGB, MARCON SR, ESPINOSA MM, et al. Fatores associados à presença de ideação suicida entre universitários. Rev. Latino-Am. Enfermagem, 2017; 25: e2878.

30. SCHNEIDERMAN N, IRONSON G, SIEGEL SD. Stress and health: psychological, behavioral, and biological determinants. Annu Rev Clin Psychol, 2005; 1: 607-628.

31. SILVA MG, COSTA ME. Vinculação aos pais e ansiedade em jovens adultos, Lisboa. Psicologia, 2005; 18(2): 9-32.

32. SOTHORNWIT J, SRISAWASDI G, SUWANNAKIN A, et al. Decreased health-related quality of life in patients with diabetic foot problems. Diabetes, Metabolic Syndrome ad Obesity, 2018; 11: 35-43.

33. SPARRENBERGER F, SANTOS I, LIMA RC. Epidemiologia do distress psicológico: estudo transversal de base populacional. Revista de Saúde Pública, 2003; 37(45): 434-439. 